\title{
EDITORIAL
}

\section{The yin and the yang of p27Kip1 as a target for cancer therapy}

\author{
B. Eymin, E. Brambilla
}

Schematically, human tumours arise because tumour cells escape from extracellular signals that physiologically monitor cellular proliferation, as well as cell death, and acquire the property to grow continuously. During tumourigenesis, many proteins that normally control the proper timing of cell growth, e.g. the cyclins, or prevent the occurrence of unappropriate apoptosis are abnormally expressed or activated, and act as typical oncogenes leading to hyperproliferation and/or protecting tumour cells from death. In contrast, proteins that normally prevent, for example, damaged cells to progress into the next phase of the cell cycle or act as apoptosis inducers, if the damage can not be repaired, are frequently inactivated in tumour cells and act as tumour suppressor genes. Therefore, loss of tumour suppressor genes associated with abnormal hyperactivity of oncogenes classically contribute to human carcinogenesis. However, affecting a clear-cut function of tumour suppressor or oncogene remains a subject of debate for certain proteins. The p27Kip1 is a member of the cyclin-dependent kinase inhibitory proteins, which also include: p21WAF1, the target gene of p53; p16INK4a, a member of the retinoblastoma (Rb) pathway; and p57Kip2. In combination with p16INK4a, it plays a major role in counteracting the activity of cyclins $D_{1}$ and $\mathrm{E}$ on $\mathrm{Rb}$ phosphorylation and subsequent $\mathrm{G}_{1}-\mathrm{S}$ transition. Therefore, it coordinates the activation of cyclin E-cdk2 with accumulation of cyclin D-cdk4 and initiates the timely exit of cells from the cell cycle in response to antimitogenic signals. Alongside its classical antiproliferative function, p27Kip1 can also promote or inhibit apoptosis depending on the context $[1,2]$. Based on these data, p27Kip1 presents most of the classical features of a tumour suppressive protein that might be lost in proliferative tumour cells.

Unlike most of the tumour suppressor genes, where loss of function responds to the classical "two-hit hypothesis" of tumor suppression (Knudson law), p27Kipl gene is not deleted (there is neither loss of heterozygosity nor homozygous deletion), and does not suffer aberrant methylation of its $5^{\prime}$-end in tumours. Rather, in mouse models, p27Kip1 was shown to be a dosage-dependent tumour suppressor, since loss of a single allele of p27Kip1 increases the frequency and decreases the latency of tumours to a level intermediate to that seen in wild-type and null counterparts [3].

In human tumours, a direct correlation between p27Kip1 protein levels and survival chances was first noted in colon cancer and later in cancers of the breast, prostate, bladder, ovary, lung and other tissues; Singerlan and PAGANO [4] give a concise review regarding $\mathrm{p} 27 \mathrm{Kip} 1$ and these cancers. The evidence that reduced $\mathrm{p} 27 \mathrm{Kip} 1$ may contribute to tumour development by either increasing the proliferation of cells or decreasing their apoptosis might explain why the loss of $\mathrm{p} 27 \mathrm{Kip} 1$ is a common marker among many different tumour

Correspondence: B. Eymin, INSERM U578 Lung Research Group, Institute Albert Bonniot, Domaine de la Merci 38706, La Tronche, Cedex, France. Fax: 33 476549509. E-mail: Beatrice.Eymin@ujf-grenoble.fr types. It also suggests that p27Kip1 maybe a valuable target for both stratifying patient risk and, perhaps, selecting treatment. However, observations that some human tumours exhibit high levels of p27Kipl makes this scheme more complex. For example, in aggressive small cell lung cancer, p27Kip1 overexpression has been suggested to protect cells from apoptosis in unfavourable microenvironments [5]. In addition, p27Kip1 might affect the efficacy of chemotherapies, as these treatments work better in proliferating rather than in growth-arrested cells. Therefore, it is necessary to keep in mind that modulating $\mathrm{p} 27 \mathrm{Kip} 1$ levels can have unforeseen consequences on therapy, depending on the cell type and the contextual clues.

In this issue, IsHII et al. [6] have studied the consequences of p27Kip1 overexpression or downregulation in human lung adenocarcinoma. The authors first show that overexpression of p27Kipl inhibits cell growth and rescues cells from death instead of inducing apoptosis. More interestingly, using short interfering (si)RNA technology, they demonstrate that neutralisation of endogenous p27Kip1 induces cell death of several nonsmall cell lung cancer cell lines without affecting their cell cycle status. Finally, after analysis of p27Kip1 subcellular localisation and phosphorylation, the authors describe the predominant cytoplasmic accumulation of endogenous p27Kip1 in these tumour cells, which might preclude it from regulating the $\mathrm{G}_{1}-\mathrm{S}$ transition. $\mathrm{p} 27 \mathrm{Kip} 1$ activity appears to be directly targeted by its mislocalisation to the cytoplasm in colon or ovarian tumours [7, 8]. In addition, the cytoplasmic p27Kip1 seems to correlate with poor long-term survival and tumour grades in breast carcinoma [9]. Mislocalisation effectively inactivates the p27Kipl inhibitory activity, as cytoplasmic p27Kip1 is partitioned from its nuclear cyclin-cdk targets. However, as suggested by IsHII et al. [6], such cytoplasmic localisation of $\mathrm{p} 27 \mathrm{Kip} 1$ could also contribute to its antiapoptotic functions. Therefore, before considering therapies, it is necessary to predict how cells in a particular tumour will respond to the accumulation or neutralisation of p27Kip1. In this context, the study by IsHII et al. [6] suggests that targeting p27Kip1 in nonsmall cell lung cancer could inhibit their growth by reducing cell viability. In contrast, in other tumour types, it will be the restoration of $\mathrm{p} 27 \mathrm{Kip} 1$ that will prevent tumour growth, either by inhibiting cellular proliferation or inducing apoptosis. Therefore, dependent upon the cancer type, the goal of therapy targeting p27Kip1 will tend to either neutralise or reintroduce it. It is the reason why comprehensive understanding of $\mathrm{p} 27 \mathrm{Kip} 1$ biology would facilitate development of such therapeutic responses in human cancer. Hence, it would be too simplistic to ascribe p27Kip1 only as a tumour suppressive protein since its anti-apoptotic function might also predict an "oncogenic" role. As a typical example of this "gain of function" are the highly agressive small cell lung cancer, which carry elevated levels of nuclear p27Kip1. Obviously, more work needs to be performed so as to decipher the real contribution of p27Kip1 in human lung-tumour growth.

In conclusion, it is now well established that the biological effects of numerous proteins might be quite different, 
sometimes even opposite, depending on the cellular model, the context and their subcellular localisation. Therefore, before designing general therapies against cancer, it is necessary to study, very carefully, the specific proteins involved in each carcinogenetic process, as well as to investigate the molecular mechanisms by which the loss or hyperactivity of such proteins contribute to the carcinogenesis. Finally, it is also of great importance to think about the fact that modulating the levels of proteins, which control essential processes, e.g. cellular proliferation and apoptosis, might interfere with the response of tumour cells to chemotherapeutic treatments as well as having unpredicted consequences.

\section{References}

1. Katayose Y, Kim M, Rakkar AN, Li Z, Cowen KH, Seth P. Promoting apoptosis: a novel activity associated with the cyclin-dependent kinase inhibitor p27. Cancer Res 1997; 57: 5441-5445.

2. Eymin B, Haugg M, Droin N, Sordet O, Dimanche-Boitrel MT, Solary E. p27Kip1 induces drug resistance by preventing apoptosis upstream of cytochrome c release and procaspase3 activation in leukemic cells. Oncogene 1999; 18: 1411-1418.
3. Fero ML, Rivkin M, Tasch M, et al. A syndrome of multiorgan hyperplasia with features of gigantism, tumorigenesis and female sterility in p27Kip1-deficient mice. Cell 1996; 85: $733-744$

4. Slingerland J, Pagano M. Regulation of the cdk inhibitor p27 and its deregulation in cancer. J Cell Physiol 2000; 183: $10-17$.

5. Masuda A, Osada H, Yatabe $\mathrm{Y}$, et al. Protective function of p27(KIP1) against apoptosis in small cell lung cancer cells in unfavorable microenvironments. Am J Pathol 2001; 158: 87-96.

6. Ishii T, Fujishiro M, Masuda M, et al. Effects of p27Kip1 on cell cycle status and viability in A549 lung adenocarcinoma cells. Eur Resp J 2004; 23: 665-670.

7. Ciaprrone M, Yamamoto H, Yao Y, et al. Localization and expression of p27Kip1 in multistage colorectal carcinogenesis. Cancer Res 1998; 58: 114-122.

8. Hurteau JA, Allison BM, Brutkiewicz SA, et al. Expression and subcellular localization of the cyclin-dependent kinase inhibitor p27(KIP1) in epithelial ovarian cancer. Gynecol Oncol 2001; 83: 292-298.

9. Viglietto $\mathrm{G}$, Motti ML, Bruni $\mathrm{P}$, et al. Cytoplasmic relocalization and inhibition of the cyclin-dependent kinase inhibitor p27Kip1 by PKB/Akt-mediated phosphorylation in breast cancer. Nat Med 2002; 8: 1136-1144. 